\title{
Utilização de extrato Artemisia vulgaris em hexano no controle in vitro de
}

\section{Rhipicephalus microplus}

\author{
Usage of the extract of Artemisia vulgaris in hexane in the in vitro control of Rhipicephalus \\ microplus
}

Utilización del extracto de Artemisia vulgaris en hexano en el control in vitro de Rhipicephalus microplus

Thiago Luiz Pereira Marques

ORCID: https://orcid.org/0000-0002-7296-9764

Universidade de Vassouras, Brasil

E-mail: marques_vet@yahoo.com.br

Gabriela Santos Freitas

ORCID: https://orcid.org/0000-0002-7073-1517

Universidade de Vassouras, Brasil

E-mail: gabriela.sanfre@gmail.com

Gabriela Barbosa Martins

ORCID: https://orcid.org/0000-0002-0099-1823

Universidade de Vassouras, Brasil

E-mail: gabrielabarbosadac@gmail.com

Letícia Meirelles Ávila

ORCID: https://orcid.org/0000-0001-7119-0639

Universidade de Vassouras, Brasil

E-mail: leticiameirellesavila@gmail.com

Karoline Alves Machado

ORCID: https://orcid.org/0000-0002-9159-8583

Universidade de Vassouras, Brasil

E-mail: karolinealvesm@gmail.com

Erica Cristina Rocha Roier

ORCID: https://orcid.org/0000-0002-1978-9254

Universidade de Vassouras, Brasil

E-mail: roier.erica@gmail.com

Bárbara Rauta de Avelar

ORCID: https://orcid.org/0000-0001-7129-8287

Universidade Federal Rural do Rio de Janeiro, Brasil

E-mail: barbararauta@gmail.com

Katherina Coumendouros

ORCID: https://orcid.org/0000-0002-9806-2618

Universidade Federal Rural do Rio de Janeiro, Brasil

E-mail: katherinac@ufrrj.br

\begin{abstract}
Resumo
As infestações por Rhiphicephalus microplus causam perdas significativas na bovinocultura brasileira provocando diversos prejuízos como, perda de peso e baixa conversão alimentar, podendo comprometer a produção de carne e leite. O extrato de plantas é utilizado como uma forma alternativa para o controle de carrapatos, visto que o uso indiscriminado de acaricidas está gerando populações de carrapatos cada vez mais resistentes. Assim, em conformidade com o exposto, o presente trabalho objetivou testar a eficiência reprodutiva in vitro de ativos extraídos em solvente apolar (hexano) da planta Artemisia vulgaris sobre o carrapato Rhipicephalus microplus. Inicialmente, para a avaliação das eficácias in vitro dos extratos da A. vulgaris, foram utilizadas teleóginas da colônia de $R$. microplus do Laboratório de Quimioterapia Experimental em Parasitologia Veterinária da UFRRJ (LQEPV), sendo separadas em grupos de 10 e submetidas ao teste de imersão em hexano na concentração de 1250 ppm, bem como controle positivo e negativo. A leitura do percentual de eclodibilidade que foi realizada para o cálculo da Eficiência Reprodutiva do Produto, foi feita através de análise de variância. Deste modo, ainda que hajam poucos trabalhos que comprovem a eficácia de A. vulgaris como um biocarrapaticida, pôde-se concluir com o presente estudo que, quando esta foi extraída em hexano demonstrou uma boa eficácia no controle in vitro de $R$. microplus.
\end{abstract}

Palavras-chave: Biocarrapaticida; Bovino; Ectoparasitas. 


\begin{abstract}
Rhiphicephalus microplus infestations cause considerable losses in Brazilian cattle farming resulting in several damage as weight loss and low feed conversion, which may affect the production of meat and milk. The extract of plants is utilized as an alternative form to control ticks, since the indiscriminate use of acaricides is causing populations of even more resistant ticks. Therefore, in conformity to the above, the present work aimed to analyze the in vitro reproductive efficiency of actives extracted in apolar solvent (hexane) of the plant Artemisia vulgaris on the tick Rhipicephalus microplus. At first, to analyze the in vitro efficacy of A. vulgaris extracts, it was used teleogines from the colony of $R$. microplus from the Laboratory of Experimental Chemotherapy in Veterinary Parasitology of UFRRJ (LQEPV), which were divided into groups of 10 and submitted to the test of immersion in hexane at a concentration of $1250 \mathrm{ppm}$, as well as positive and negative control. The measurement of the percentage of hatchability, which was performed to calculate the Reproductive Efficiency of the Product, was done using variance analysis. In this way, despite the fact that there are few studies that prove the efficacy of A. vulgaris as a biocarpaticide, it could be concluded with the present study that when it was extracted in hexane it demonstrated a good efficiency in the in vitro control of $R$. microplus.
\end{abstract}

Keywords: Biocarrapaticide; Bovine; Ectoparasites.

\title{
Resumen
}

Las infestaciones por Rhiphicephalus microplus causan importantes perjuicios en la ganadería brasileña, provocando varias dificultades como la pérdida de peso y la baja conversión alimenticia, lo que puede comprometer la producción de carne y leche. El extracto de plantas se utiliza como una forma alternativa de controlar las garrapatas, ya que el uso indiscriminado de acaricidas está generando poblaciones de garrapatas cada vez más resistentes. Así, de acuerdo con lo anterior, el presente trabajo tuvo como objetivo comprobar la eficacia reproductiva in vitro de activos extraídos en disolvente apolar (hexano) de la planta Artemisia vulgaris sobre la garrapata Rhipicephalus microplus. Inicialmente, para evaluar la eficacia in vitro de los extractos de A. vulgaris, se utilizaron teleoginos de la colonia de $R$. microplus del Laboratorio de Quimioterapia Experimental en Parasitología Veterinaria de la UFRRJ (LQEPV), separados en grupos de 10 y sometidos a la prueba de inmersión en hexano a una concentración de 1250 ppm, así como el control positivo y negativo. La lectura del porcentaje de incubabilidad que se realizó para el cálculo de la Eficiencia Reproductiva del Producto se hizo a través del análisis de varianza. Así pues, aunque hay pocos estudios que demuestren la eficacia de A. vulgaris como biocarpaticida, se puede concluir con el presente estudio que cuando se extrajo en hexano demostró una buena eficacia en el control in vitro de $R$. microplus.

Palabras clave: Biocarrapaticida; Bovino; Ectoparásitos.

\section{Introdução}

Segundo dados divulgados em 2020 pela Associação Brasileira das Indústrias Exportadoras de Carnes (ABIEC), o Brasil possui 213,68 milhões de cabeças de gado, ocupando o primeiro lugar no ranking mundial de exportação de carne bovina, com cerca de 2,49 milhões de toneladas equivalente a carcaça. Além disso, é o quinto maior produtor mundial de leite, com uma produção estimada em 34,6 milhões de toneladas só em 2019 (Embrapa, 2020).

O carrapato Rhipicephalus microplus, parasita bovinos, onde afeta aspectos econômicos e sanitários da produção de carne, leite e couro (Cognini et al., 2020). O R. microplus é encontrado em regiões de clima tropical e subtropical, pois esse clima é favorável a reprodução desse ectoparasito (Ali et al., 2016).

No que concerne a bovinocultura, há uma relação direta e proporcional entre o aumento da população de carrapatos e a incidência da Tristeza Parasitária dos Bovinos (TPB), que é considerada um complexo de doenças compreendendo duas enfermidades bem conhecidas: a babesiose, causada pelos protozoários Babesia bigemina e Babesia bovis, e a anaplasmose causada pela Anaplasma marginale (Guedes Júnior et al., 2008).

Além do potencial de transmitir agentes patogênicos, essa espécie de carrapato, quando em altas infestações, causa diversas lesões na pele do animal possibilitando assim que bactérias oportunistas venham a causar infecções nos bovinos (infecções secundárias). (Reck et al., 2014).

Os danos causados podem ainda ser atribuídos à perda de peso dos animais e também à redução da produção de leite, decorrentes da intensa espoliação sanguínea e irritabilidade provocada pelos carrapatos em função de sua picada. A morte de animais, como consequência das altas infestações por carrapatos, também é uma triste realidade comumente presente no sistema de produção de bovinos (Andreotti et al., 2019) 
Para o tratamento de tal ectoparasitose, rotineiramente são utilizados carrapaticidas sintéticos, entretanto tais substâncias podem potencialmente causar não só impactos ambientais, como riscos de contaminação de carne e de leite. Além disso, tem-se observado resistência por parte do R. microplus à maioria dos acaricidas, principalmente os organofosforados, piretróides sintéticos, amitraz, ivermectina e fipronil (Rayal et al, 2018).

Uma forma alternativa para o controle de carrapatos é a utilização de extratos de plantas. Essa prática é antiga e são utilizados popularmente no controle de ectoparasitos há muitos séculos por chineses, indianos, egípcios e indígenas, porém vem recebendo mais atenção dos pesquisadores atualmente e com isso está sendo realizados alguns estudos no Brasil onde, uma variedade de espécies está sendo pesquisadas com intuito de se avaliar seu efeito carrapaticida. (Santos \& Vogel, 2012). Alguns extratos vegetais já possuem ação pesticida comprovada, que são ativos variados provenientes do metabolismo secundário das plantas. Esses extratos apresentam como benefícios segurança, baixo custo, baixa toxicidade, contribuem para reduzir os impactos ambientais e tendem a fazer menos mal ao homem, o que justifica o seu uso (Moreira et al., 2007; Bagavan et al., 2009).

A Artemisia vulgaris possui origem na Ásia e Europa, mas está presente em quase todo o globo, devido a característica de invasora, sendo assim pouco exigente quanto a clima e solo, e por isso pode ser encontrada em campos cultivados, jardins, pastagens e margens de estradas (Panizza, 1997; Oliveira et al., 2009). Também conhecida pelo nome científico Artemisia verlotorum e outros nomes populares como: artemigem, artemígio, flor-de-são-joão, anador, artemísiacomum, artemísia-vulgar, erva-de-são-joão, losna-brava, artemísia-verdadeira, absintoselvagem e isopo-santo (Oliveira, 2009).

Panizza (1997) e Oliveira et al., (2009) cita os seus constituintes, sendo: óleos essenciais ricos em terpenos (cineol e tuiona), flavonóides, taninos, saponinas, resinas, artemisina e princípios amargos. Dentre esses compostos com relação ao potencial acaricida já foi citado na utilização de extratos metanólicos e etanólicos, obtidos de folhas de espécies do gênero Artemisia, sendo a seguinte relação: A. abrotanum e A. absintum por conter esquiterpenos (Geissman, 1970).

Todas as partes da A. vulgaris têm sido usadas por todo o mundo na medicina popular durante séculos. Possui diversas ações no organismo, como atividade analgésica, anti-helmíntica, antibacteriana, antiflatulenta, antifúngica, antireumática, antisséptica, afrodisíaca, estimulante do apetite, estimulante da bile, depressora do SNC, contra-irritante, diaforética, digestiva, diurética, emética, expectorante, hemostática, laxativa, sedativa, estimulante do útero e vasodilatadora uterina (Fetrow \& Ávila, 2000), anti-inflamatória, antiespasmódicos e anticonvulsivos, age também em casos de dispepsia, astenia, dores reumáticas, febres, e anemias (TIGNO et al., 2000). Também é recomendada para uso externo contra escaras, feridas, piolhos, lêndeas e Ctenocephalies felis felis (Oliveira et al., 2009; Souza, 2013). O presente trabalho objetivou testar a eficiência reprodutiva de ativos extraídos em solvente apolar (hexano) da planta Artemisia vulgaris sobre o controle in vitro de Rhipicephalus microplus.

\section{Metodologia}

O presente trabalho está incluído no processo de nº. 4667181218 do CEUA (Comitê de Ética no Uso de Animais) da Universidade Federal Rural do Rio de Janeiro referente aos procedimentos adotados na colônia de R. microplus do Laboratório de Quimioterapia Experimental em Parasitologia Veterinária da UFRRJ. O suporte metodológico para realização da pesquisa foi segundo Estrela (2018).

A amostra de Artemisia vulgaris foi coletada no horto de plantas medicinais da Universidade de Vassouras-RJ, no período de abril de 2019. Foi obtido um total de aproximadamente $1 \mathrm{Kg}$ da planta para a formulação do extrato, sendo conduzida ao departamento de farmácia (DCFAR) da Universidade Federal Rural do Rio de Janeiro (UFRRJ), onde foi realizada inicialmente uma triagem com o intuito de retirar plantas invasoras diferentes da escolhida e fazer a separação de 
partes do vegetal com menor potencial de extração. Esta foi então posta em bandejas e acondicionada em estufa de secagem para posterior preparo do extrato.

A preparação do extrato também foi realizada no DCFAR da UFRRJ. Já a partição posterior foi feita no departamento de farmácia do Laboratório de Quimioterapia Experimental em Parasitologia Veterinária da UFRRJ (LQPV). Após a obtenção do extrato bruto através do metanol (polaridade 5,1) que possibilita a extração de um maior número de compostos, este foi encaminhado para a partição posterior líquido-líquido, com hexano, solvente semi-polar (polaridade 0).

Para a obtenção dos R. microplus foram utilizados bezerros mantidos em baias individuais com alimentação e água ad libitum, sem contato recente com carrapaticidas químicos, provenientes da colônia do Laboratório de Quimioterapia Experimental em Parasitologia Veterinária da UFRRJ. Esses foram infestados artificialmente com larvas de R. microplus na região dorsal, para realização da fase parasitária. Após 21 dias, foram coletadas teleóginas do piso das baias, que em seguida foram levadas ao laboratório de parasitologia, para serem usadas no presente estudo.

Os testes in vitro foram realizados no Laboratório de Quimioterapia Experimental em Parasitologia Veterinária da UFRRJ. As teleóginas recebidas no laboratório foram lavadas em água corrente, e posteriormente foram submetidas à secagem com papel absorvente, então as que possuíam perfeito estado físico foram selecionadas e separadas em grupos de 10 teleóginas por tratamento.

Para os testes a eficácia da Artemísia vulgaris foi feito um ensaio a partir dos extratos obtido do hexano 5 gotas de tween na solução mãe. Assim, o ensaio foi realizado através da composição de 4 grupos com 10 teleóginas em cada. O primeiro com extrato de A. vulgaris em concentração de 1250ppm, mais um grupo controle positivo (testadas com fipronil), um grupo controle negativo (testadas com água) e um grupo placebo (testadas com solvente sem o extrato).

Após a separação em grupos, as teleóginas foram imersas em $20 \mathrm{ml}$ da solução do tratamento, grupo controle positivo (fipronil), negativo (água) e placebo (solventes) por 5 minutos. Logo após a imersão, o excesso dos produtos foi retirado com o auxílio de peneira e papel absorvente, e assim as teleóginas foram pesadas individualmente em balança analítica, baseado na técnica descrita por Drummond et al. (1973). Após a pesagem elas foram acondicionadas em placa de petri descartável, devidamente identificada com data e número referente a cada teleóginas totalizando 10 teleóginas por placa, fixadas utilizando fita adesiva dupla face. As placas de petri foram incubadas em câmara climatizada com demanda biológica de oxigênio (B.O.D) a $27^{\circ} \mathrm{C}$ de temperatura e $80 \%$ de umidade relativa, por 21 dias, para a oviposição. Depois da realização das posturas, essas foram pesadas em balanças analíticas com o intuito de determinar as eficácias. As posturas foram então transferidas para seringas fechadas com algodão, e após serem devidamente identificadas como as placas de petri retornaram à estufa, nas mesmas condições de umidade e temperatura anteriormente citadas até a eclosão das larvas (14 dias).

A leitura do percentual de eclodibilidade foi feito com o auxílio de um estereoscópio, e então foram calculadas a Eficiência Reprodutiva (ER) e a Eficiência do Produto (EP), utilizando a fórmula já descrita por Drummond et al. (1973), para calcular a eficácia da A. vulgaris sobre fêmeas ingurgitadas de R. microplus. As equações utilizadas para cálculo de eficiência reprodutiva, Equação 1 e Eficácia do Produto, Equação 2 estão representadas abaixo.

\section{Equação 1}

$$
E R=\frac{\text { Peso dos ovos } X \% \text { de eclosão } X 20.000 *}{\text { Peso das teleó ginas }}
$$

Legenda: ER = Eficácia Reprodutiva; * = Número de larvas por 1g de ovos. 


\section{Equação 2 \\ $E P=\frac{(E R \text { do } G \text { controle }-E R \text { do } G \text { tratado }) X 100}{E R \text { do } G \text { controle }}$}

Legenda: EP = Eficácia do produto

A base para eficácia de cada produto foi calculada através da ER dos grupos controle negativo. Segundo a normativa 48/1997 do Ministério da Agricultura, Pecuária e Abastecimento, os carrapaticidas devem obter 95\% de eficiência para ser considerado um produto eficaz. Em distinção ao citado pelo MAPA, Aurnheimer et al. em 2007 considerou valores mínimos de $75 \%$ para validar um produto eficiente. Todas as análises foram calculadas considerando o intervalo de confiança de $95 \%$ (p $\leq 0,05)$.

\section{Resultados}

O teste realizado para determinar a eficácia do extrato de Artemisia vulgaris foi eficiente no controle do R. microplus nos ativos extraído em hexano com concentração de $1250 \mathrm{ppm}$, ficando superior a norma de avaliação de ação carrapaticida que considera eficiente os resultados acima de $95 \%$ reforçando para este extrato a suspeita de ação acaricida.

A Tabela 1 demonstra os resultados do extrato de Artemísia vulgaris em Hexano. A média dos pesos da postura no grupo controle negativo e na concentração de $1250 \mathrm{ppm}$ foi respectivamente 0,099 e $0,008 \mathrm{~g}$, e o grupo controle positivo não realizou postura. Ao analisar as eclodibilidades a concentração de $1250 \mathrm{ppm}$, obteve um resultado inferior a 10\%, apresentando 7,3. Além disso com relação ao percentual de eficácia apresentou eficiência superior a 95\% uma vez que determinou 98,1\%.

Tabela 1: Análise da variância para determinação do peso médio das teleóginas, das posturas, percentual médio de eclosão, percentual médio de mortalidade, média da eficiência reprodutiva. Teste de imersão de adultos de R. microplus, em concentração de 1250 ppm do extrato de Artemisia vulgaris em em Hexano.

\begin{tabular}{cccccc}
$\begin{array}{c}\text { Concentração } \\
{[\mathrm{ppm}]}\end{array}$ & $\begin{array}{c}\text { Média Peso } \\
\text { Teleógina }\end{array}$ & $\begin{array}{c}\text { Média Peso } \\
\text { Postura }\end{array}$ & $\begin{array}{c}\text { Média }(\%) \\
\text { Eclodibilidade }\end{array}$ & $\begin{array}{c}\text { Média }(\%) \\
\text { Mortalidade }\end{array}$ & $\begin{array}{c}\text { Eficácia } \\
(\%)\end{array}$ \\
\hline $\begin{array}{c}\text { Controle } \\
\text { negativo } \\
1250\end{array}$ & $0,240 \mathrm{a} \pm 0,017$ & $0,099 \mathrm{a} \pm 0,050$ & $78,6 \pm 31,2$ & $21,4 \pm 31,2$ & -- \\
$\begin{array}{c}\text { Controle } \\
\text { positivo }\end{array}$ & $0,248 \mathrm{a} \pm 0,041$ & $0,008 \mathrm{~b} \pm 0,020$ & $7,3 \pm 15,8$ & $92,7 \pm 15,8$ & 98,1 \\
\hline
\end{tabular}

Fonte: Autores.

\section{Discussão}

Mcgaw e Eloff em 2008 ressaltaram a importância de validar a utilização de plantas através do estabelecimento de doses corretas, a fim de melhor via de aplicação e garantir a ausência de toxicidade. Almeida et al. (2006) ao aplicar um questionário sobre etnoveterinária junto aos estudantes de medicina veterinária em Mossoro, Rio Grande do Norte pode demonstrar que os próprios estudantes desconhecem este termo demonstrando que ainda existe poucas iniciativas de se identificar plantas com interesse médico veterinário, ressaltando a importância de estudos como esse que permitem que haja registro da utilização de plantas para tratar as principais doenças que acometem os animais. Aurnheimer et al. (2012) cita 75\% 
como o menor percentual para considerar um produto acaricida eficiente, sendo assim, no presente estudo a A. vulgaris $(98,1 \%)$, seguindo a metodologia utilizada, apresenta esse potencial de eficiência. Além de estar acima do preconizado pela OMS (75\%) e no decreto 48 de 1997 (95\%) do MAPA. Ao avaliar o potencial acaricida foi possível observar redução significativa na média de peso da postura quando o tween foi acrescentado, corroborando com Perinotto (2012) ao afirmar que a redução da oviposição é considerada como um dos pontos mais importantes para o controle de carrapatos, já que a redução do número total de ovos, em conjunto com a viabilidade dos mesmos, irá comprometer as gerações seguintes e reduzirá a população de carrapatos no ambiente. O tween é um produto químico que promove redução da tensão interfacial entre os componentes da formulação, permitindo a obtenção de emulsões estáveis tal como descreve o fabricante (MAPRIC®) o que pode elucidar os resultados obtidos no presente trabalho ressaltando assim a importância deste composto no preparo de extratos de A. vulgaris.

Molan et al. (2003) e Sprenger et al. (2015) acreditam que o fato de os taninos agirem reduzindo a oviposição e diminuindo a fertilidade das fêmeas adultas de nematodas, pode agir de forma similar em ectoparasito explicando a redução na oviposição das teleóginas de $R$. microplus, após serem tratadas com a A. vulgaris, uma vez, o tanino está presente na composição química desta planta (Panizza, 1997; Oliveira, et al., 2009). Além do tanino outra substância significativamente predominante nesta planta é uma lactona sesquiterpêniaca conhecida como artemisina (Moreira, 1985). A essa substância é atribuído o potencial parasiticida uma vez que apresenta eficiência comprovada no controle de bezouros agindo como inseticida e leishmanicida, sendo esses resultados observados em extratos ricos em artemisina obtidos da planta Tithonia diversifolia da mesma família da A. vulgaris (Ambrosio, 2007). Esses relatos fortalecem a hipótese de que a substância que promoveu a eficiência nos extrados de A. vulgaris utilizados no presente estudo tenha sido a artemisina. Apesar da ampla descrição contra diferentes parasitos, não houve na literatura ação da A. vulgaris contra R. microplus. Porém, a ação contra este parasito é relatada utilizando extratos etanólicos de А. аппиа citando também a artemisina como principal agente, devido sua estrutura química peculiar que possui alta estabilidade térmica, baixa toxicidade e boa eficiência contra parasitos resistentes a outras drogas (Meshnick, 1996).

A utilização da A. vulgaris no presente estudo visa o uso tópico pelo próprio contato com o parasito, porém Chagas et al. (2011) já descreveu a apresentação oral da A. vulgaris contra nematóides gastrointestinais, trematódeos uma vez que estudos evidenciam uma boa eficiência quando a Artemísia reage com a fração heme do sangue fato utilizado para justificar a não eficiência da Artemísia annua sobre larvas de R. microplus sugerindo assim uma melhor eficiência no produto oral em comparação com o tópico.

\section{Conclusão}

Embora sejam poucos trabalhos que comprovem a eficácia reprodutiva de Artemisia vulgaris como um biocarrapaticida, no presente estudo quando essa foi extraída em hexano demonstrou uma boa eficácia no controle in vitro de Rhipicephalus microplus. O maior desafio foi promover a solubilização dos extratos, no entanto foi possível obter resultados satisfatórios. Uma vez que o uso de plantas medicinais em Medicina Veterinária é comum, se faz importante após a validação a elaboração de cartilhas visando a orientação dos proprietários e produtores. Além disso, é necessário trabalhos futuros que façam testes de segurança para realização de testes in vivo.

\section{Referências}

Ali, A., Parizi, L. F., Ferreira, B. R., \& Vaz Junior, I. D. S. (2016). A revision of two distinct species of Rhipicephalus: R. microplus and R. australis. Ciência Rural, 46(7), 1240-1248.

Almeida, K. A., Costa Freitas, F. L., \& Pereira, T. F. C. (2006). Etnoveterinária: a fitoterapia na visão do futuro profissional veterinário. Revista verde de agroecologia e desenvolvimento sustentável, 1(1). 
Ambrosio, S. R.; Toledo, J. S.; Toledo, T. C. I.; Cerri, D. G.; Lopes, W.; Cruz, A. K., \& Costa, F. B. (2007). Atividade leishmanicida de lactonas sesquiterpênicas de Tithonia diversifolia (Asteraceae). In: Reunião Anual Da Sociedade Brasileira De Química - SBQ, 30, Águas de Lindóia. Anais [...] Águas de Lindóia - SP.

Andreotti, R.; Garcia, M. V. \& Koller W.W. (2019). Controle estratégico dos carrapatos nos Bovinos. In: Carrapatos na cadeia produtiva de bovinos. Brasília, DF, 123-133.

Aurnheimer, R. C., Pereira, M. A. V., Vita, G. F., \& Damas, S. L. (2012). Eficácia in vitro de Ruta graveolens, nas formas fitoterápica e homeopática, para o controle de carrapatos. Ars Vet., 28(2) 122-127.

Bagavan, A., Kamaraj, C., Elango, G., Zahir, A. A., \& Rahuman, A. A. (2009). Adulticidal and larvicidal efficacy of some medicinal plant extracts against tick, fluke and mosquitoes. Veterinary parasitology, 166(3-4), 286-292.

Brasil. Ministério da Agricultura e do Abastecimento. (1997) Secretaria de Defesa Agropecuária. Portaria n. 48 de 12 de maio de 1997. Regulamento técnico para licenciamento e/ou renovação de licença de produtos antiparasitários de uso veterinário.

Chagas, A. C. S, Georgetti, C. S., Carvalho, C. O., Oliveira, M. C. S., Rodrigues, R. A., Foglio, M. A., \& Magalhães, P. M. (2011). In vitro activity of Artemisia annua L (Asteraceae) extracts against Rhipicephalus (Boophilus) microplus. Revista Brasileira de Parasitologia Veterinária, 20(1), 31-35

Cognini, E. F. T., Vargas, G. P., Fiorio, M. S., Wortmann, B. B., Oliveira, L. R. S., Rosa, R. L., Souza, E. M., Santi, L., \& Silva, W. O. (2020). Métodos alternativos e sustentáveis de controle do carrapato bovino Rhipicephalus microplus. Revista Liberato, 20(34), $27-37$.

Drummond, R. E. A., Ernst, S. E., Trevino, J. L., Gladney, W. J., \& Graham, O. H. (1973). Boophilus annulatus and B. microplus: laboratory tests of insecticides. Journal of economic entomology, 66(1), 130-133.

Empresa Brasileira de Pesquisa Agropecuária (Embrapa). (2020). Anuário Leite. Disponível em: https://www.embrapa.br/busca-de-publicacoes//publicacao/1124722/anuario-leite-2020-leite-de-vacas-felizes

Estrela, C. (2018). Metodologia Científica: Ciência, Ensino, Pesquisa. Editora Artes Médicas.

Fetrow, C. W., \& Avila, J. R. (2000). Manual de medicina alternativa para o profissional. Guanabara Koogan.

Geissman, T. A. (1970). Sesquiterpene lactones of Artemisia-A. verlotorum and A. vulgaris. Phytochemistry, 9(11), $2377-2381$.

Guedes, D. S., Jr., Araújo, F. R., Silva, F. J. M., Rangel, C. P., Barbosa, J. D., Neto, \& Fonseca, A. H. (2008). Frequency of antibodies to Babesia bigemina, B. bovis, Anaplasma marginale, Trypanosoma vivax and Borrelia burdgorferi in cattle from the northeastern region of the state of Pará, Brazil. Revista Brasileira de Parasitologia Veterinária, 17(2), 105-109.

McGaw, L. J., \& Eloff, J. N. (2008). Ethnoveterinary use of southern African plants and scientific evaluation of their medicinal properties. Journal of Ethnopharmacology, 119(3), 559-574.

Meshnick, S. R., Jefford, C. W., Posner, G. H., Avery, M. A., \& Peters, W. (1996). Second-generation antimalarial endoperoxides. Parasitology Today, 12(2), 79-82.

Estrela, C. (2018). Metodologia Científica: Ciência, Ensino, Pesquisa. Editora Artes Médicas.

Molan, A. L., Meagher, L. P., Spencer, P. A., \& Sivakumaran, S. (2003). Effect of flavan-3-ols on in vitro egg hatching, larval development and viability of infective larvae of Trichostrongylus colubriformis. International Journal for Parasitology, 33(14), 1691-1698.

Moreira, F. (1985). As plantas que curam. São Paulo: Hemus, 256 p.

Moreira, M. D., Picanço, M. C., Silva, E. D., Moreno, S. C., Martins, J. C., Venzon, M., \& Pallini, A. (2006). Uso de inseticidas botânicos no controle de pragas. Controle alternativo de pragas e doenças. Viçosa: EPAMIG/CTZM, 89-120.

Oliveira, M. I., Castro, E. M., Costa, L. C. B., \& Oliveira, C. (2009). Características biométricas, anatômicas e fisiológicas de Artemisia vulgaris L. cultivada sob telas coloridas. Revista Brasileira de Plantas Medicinais, 11(1), 56-62.

Panizza, S. (1997). Plantas que curam: cheiro do mato. (19a ed.), IBRASA. 279 p.

Perinotto, W. M. S., da Costa Angelo, I., Gôlo, P. S., Camargo, M. G., de Sá, F. A., de Oliveira Monteiro, C. M., \& Bittencourt, V. R. E. P. (2012). Eficiência da formulação comercial de Beauveria bassiana no controle de Rhipicephalus microplus em condições laboratoriais. Brazilian Journal of Veterinary Medicine, 34(Supl. 1), 95-101.

Raynal, J. T., Borges, A. A., Bastos, B. L., Bahiense, T. C., Meyer, R., \& Portela, R. W. (2018). Avaliação da eficiência de acaricidas sobre Rhipicephalus (Boophilus) microplus no estado da Bahia. PUBVET, 12, 133.

Reck, J., Marks, F. S, Rodrigues, R. O, Souza, U. A, Webster, A., Leite, R. C, \& Martins, J. R (2014). A infestação por carrapatos Rhipicephalus microplus aumenta o risco de miíase causada por Cochliomyia hominivorax em bovinos? Medicina veterinária preventiva , 113 (1), $59-62$.

Santos, F. D., \& Vogel, F. S. F. (2012). Avaliação in vitro da ação do óleo essencial de capim limão (Cymbopogon citratus) sobre o carrapato bovino Rhipicephalus (Boophilus) microplus. Revista Brasileira de Plantas Medicinais, 14(4), 712-716.

Souza, M. C. L. Avaliação da ação inseticida e de repelência de Artemisia vulgaris L. sobre Ctenocephalides Felis Felis (Bouché, 1835) (Siphonaptera, Pulicidae) em condições de laboratório. 2013. Monografia (Especialista em Entomologia Urbana) - Universidade Estadual Paulista Júlio De Mesquita Filho, 2013. 
Research, Society and Development, v. 10, n. 13, e129101320759, 2021

(CC BY 4.0) | ISSN 2525-3409 | DOI: http://dx.doi.org/10.33448/rsd-v10i13.20759

Sprenger, L. K., Buzatti, A., Campestrini, L. H., Yamassaki, F. T., Maurer, J. B. B., Baggio, S. F. Z., \& Molento, M. B. (2015). Atividade ovicida e larvicida do extrato hidroalcoólico de Artemisia annua sobre parasitas gastrintestinais de bovinos. Arquivo Brasileiro de Medicina Veterinária e Zootecnia, 67(1), 2531.

Tigno, X. T., de Guzman, F., Flora, A. M., \& Theresa, V. (2000). Phytochemical analysis and hemodynamic actions of Artemisia vulgaris L. Clinical hemorheology and microcirculation, 23(2,3,4), 167-175. 